\title{
Karl Kraus lettore di Schopenhauer ${ }^{1}$
}

\author{
Karl Kraus leitor de Schopenhauer
}

\author{
Maria Vitale \\ Doutora em Filosofia pela Università degli Studi del Salento (Lecce-Itália), \\ Membro do "Centro interdipartimentale di ricerca su Arthur Schopenhauer e la sua scuola". \\ E-mail: mariavitale@live.it
}

\begin{abstract}
Riassunto: A partire da un articolo di Bernard Adamy pubblicato nello «Schopenhauer Jahrbuch» del 1985 intitolato Schopenhauer Rezeption bei Karl Kraus, il presente contributo intende mettere in evidenza gli aspetti che avvicinano il pensiero Kraus a Schopenhauer. Infatti, ispirandosi alle tesi schopenhaueriane sullo stile, con una pungente satira, nella sua «Die Fackel» Kraus attacca le istituzioni politiche e culturali della Vienna asburgica, denunciando soprattutto gli orrori della prima guerra mondiale e la corruzione della stampa, e contemporaneamente offre il suo punto di vista sullo stile e la teoria linguistica.
\end{abstract}

Parole chiave: Kraus; Teoria linguistica; Die Fackel.

Resumo: A partir de um artigo de Bernard Adamy, publicado no Schopenhauer-Jahrbuch de 1985 e intitulado Schopenhauer Rezeption bei Karl Kraus, o presente texto pretende pôr em evidência os aspectos que aproximam o pensamento de Kraus ao de Schopenhauer. Com efeito, inspirando-se nas teses schopenhauerianas sobre o estilo, na sua Die Fackel Kraus ataca, com uma sátira pungente, as instituições políticas e culturais da Viena absburga. Com isso, o autor denuncia sobretudo os horrores da Primeira Guerra Mundial e a corrupção das publicações; e apresenta, assim, o seu ponto de vista sobre o estilo e sobre a teoria linguística.

Palavras-chave: Kraus; Teoria linguística; Die Fackel.

In un articolo della «Die Fackel» del gennaio del 1919, Karl Kraus si definì «un osservatore scettico delle mascherate umane ${ }^{2} \gg$. Grattare via «la vernice lucente di quel quadro fastoso ${ }^{3} »$ che era la Vienna asburgica dei primi del Novecento, fu il compito al quale il giornalista austriaco dedicò l'intera vita. La corruzione della stampa, gli orrori della prima guerra mondiale e l'ascesa del nazionalsocialismo furono solo alcuni dei temi affrontati dalla rivista dalla copertina rossa, che tutti leggevano ma che nessuno aveva il coraggio di nominare, tanto che il giornale diventò presto uno «specchio di vita viennese ${ }^{4}$ ». Così decidendo di combattere la stampa con la stampa stessa, Kraus condusse quella che è stata definita la sua «appassionata lotta contra la stampa ${ }^{5} »$. Illuminando con la

\footnotetext{
${ }^{1}$ Il presente saggio è stato da me presentato in qualità di relatore al XXXI Seminario Internazionale di Stud italo-tedeschi sul tema Mito e realtà dell'impero austroungarico, organizzato dall'Accademia di Studi Italo-Tedeschi di Merano in collaborazione con le Università del Salento e di Friburgo i. B., tenutosi a Merano dal 27 al 29 aprile 2009.

${ }^{2}$ E. Timms, La Vienna di Karl Kraus, Bologna, Il Mulino 1986, cit., p. 449. Cfr l'articolo della «Die Fackel» del gennaio 1919 , p. 82.

${ }^{3}$ K. Kraus, Aforismi, a cura di P. Sorge, Firenze, Sansoni Editore 1992, cit., introduzione.

${ }^{4}$ Ibidem.

${ }^{5}$ E. Fischer, Karl Kraus, Robert Musil, Franz Kafka, presentazione di L. Lombardo Radice, Firenze, La Nuova Italia 1974, cit., p. 6.
} 
sua fiaccola la società della sua epoca, fece emerge, con assoluta nitidezza le poche virtù ed i molti vizi. Ma l'altro tema affrontato apertamente da Kraus, che rappresenta un primo punto di contatto con Schopenhauer, è il problema dello stile e della teoria linguistica.

Nei Parerga e Paralipomena del 1851, opera con la quale ebbe inizio la fortuna tedesca ed europea di Arthur Schopenhauer, il filosofo aveva dichiarato che:

È proprio la capacità di chiarezza che conferisce alla lingua il suo valore ${ }^{6}$.

Questa affermazione sta a testimoniare un principio stilistico al quale Schopenhauer rimase sempre fedele e, al contempo, riflette l'atteggiamento del filosofo che in questo modo intese sempre mostrare la sua onestà intellettuale. Ed i Parerga, che Schopenhauer considerava «scritti minori che si aggiungono alle opere sistematiche ${ }^{7} »$ erano stati elaborati proprio con l'intenzione di offrire «ancora molti chiarimenti ${ }^{8} \gg$, sul suo sistema filosofico esposto nel Mondo, ma in una forma più divulgativa e rivolta ad un pubblico più ampio.

Nei tre scritti dedicati al linguaggio, vale a dire Sul mestiere dello scrittore e sullo stile ${ }^{9}$, Del leggere e dei libri $^{10} \mathrm{e}$, infine, Della lingua e delle parole ${ }^{11}$, Schopenhauer dichiara:

L'oscurità, la mancanza di chiarezza nell'espressione è sempre e dovunque un sintomo assai brutto. Poiché in novantanove casi su cento essa deriva dalla mancanza di chiarezza nel pensiero, che a sua volta deriva quasi sempre dalla sua originaria incongruenza, inconsistenza e dunque inesattezza ${ }^{12}$.

Un esempio di scrittura fumosa e, a detta del filosofo, incongruente sarebbe proprio quello dei tre idealisti Fichte, Schelling ed Hegel, che egli giudica abili sofisti, il cui unico talento si esprimerebbe nella capacità di trarre in inganno il lettore. Si tratterebbe, cioè, di quella che Schopenhauer ha chiamato

La maschera più resistente a quella dell'incomprensibilità, sebbene ciò avvenga solo in Germania, introdotta da Fichte, essa è stata perfezionata da Schelling e ha raggiunto il suo vertice con Hegel: e sempre con un esito fortunatissimo [...]. Eppure non vi è nulla di più facile che scrivere in modo che nessuno capisca e nulla di più difficile che

\footnotetext{
${ }^{6}$ A. Schopenhauer, Sul mestiere dello scrittore e sullo stile, trad. it. E. Amendola Kuhn, con una nota di F. Volpi, Milano, Adelphi, 1993, cit., p.67.

${ }^{7}$ A. Schopenhauer, Parerga e paralipomena, a cura di G. Colli, Milano, Adelphi, 1981, tomo I, cit., p.14

${ }^{8}$ Ibidem.

${ }^{9}$ Schopenhauer, Sul mestiere dello scrittore e sullo stile, trad. it. E. Amendola Kuhn, con una nota di F. Volpi, Milano, Adelphi, 1993, cit., pp. 17- 106.

${ }^{10}$ Id., cit.,pp. $107-125$.

${ }^{11} I d$., cit., pp.125- 149.

${ }^{12}$ Id., cit., p. 54.
} 
esprimere pensieri significativi in modo che ognuno debba comprenderli ${ }^{13}$.

Ed è il già citato capitolo dei Parerga e paralipomena, «Del leggere e dei libri», che fornisce il materiale letterario al quale $\mathrm{Karl} \mathrm{Kraus}^{14}$ fa riferimento nel criticare il malcostume letterario del suo tempo.

Pur non riferendosi all'analisi antropologica schopenhaueriana e non condividendo il pessimismo metafisico del filosofo del Mondo e la sua dimensione filosofica, Karl Kraus considerava lo stile schopenhaueriano un modello linguistico da seguire e apprezzandone la chiarezza nello stile lo considerava un esempio di onestà intellettuale.

Ciò che Kraus intendeva far proprio e sviluppare di Schopenhauer erano soprattutto le osservazioni di quest'ultimo intorno all'estetica. Così egli elogiava le riflessioni di Schopenhauer in merito all'argomento, in un articolo della sua rivista «Die Fackel»:

Tra tutte le definizioni, a partire da Schiller e Spencer, me ne appare solo una significativa ed illuminante: l'interpretazione di Schopenhauer dell'arte come oggettivazione della Volontà ${ }^{15}$.

Come è noto, infatti, nel Mondo come volontà e rappresentazione, Schopenhauer esponeva, con una celebre metafora, l'idea per cui la vita non è che un pendolo che oscilla tra il dolore e la noia, per sottolineare lo stato di perenne bisogno in cui l'uomo si trova, ovvero lo stato di completa sottomissione alla Volontà di vita universale. Essa, infatti, condanna l'uomo a desiderare continuamente senza mai poter appagare il suo desiderio. Fra le vie di liberazione possibili dalla Volontà universale, Schopenhauer indicava quella principale della negazione della Volontà attraverso il nirvana, ma anche l'esperienza estetica, ovvero quella della contemplazione delle idee: in questa maniera, il genio, l'unico in grado di poter cogliere le idee, si sarebbe mutato nel «puro occhio del mondo ${ }^{16}$ », obliando così se stesso come essere determinato e quindi sottoposto alla signoria della Volontà di vita.

Come Schopenhauer, anche Kraus era dell'avviso che l'opera d'arte non dovesse avere nessuno scopo didattico-educativo, ma che essa fosse esclusiva prerogativa del genio. Quindi, con Schopenhauer, Kraus ammetteva un primato del genio in senso romantico, e quindi individualistico. Kraus era, fra l'altro, dell'opinione che l'arte del suo tempo fosse in declino, tanto da essere derubricata

\footnotetext{
${ }^{13}$ Id., cit., p. 46.

${ }^{14}$ Cfr. B. Adamy, Schopenhauer’s Rezeption bei Karl Kraus, in « Schopenhauer Jahrbuch », 1985, cit., pp. $85-102$.

${ }^{15}$ Id., cit., p. 100.

${ }^{16}$ A. Schopenhauer, Il mondo come volontà e rappresentazione, trad., it. S. Giametta, Milano, Bompiani, 2006, cit.,p. 377.
} 
allo status di vano passatempo ${ }^{17}$ e semplice distrazione ricreativa.

Inoltre, sempre sulla falsariga delle riflessioni di Schopenhauer sull'estetica, Kraus sosteneva, che la lingua era «un' opera d'arte completa ${ }^{18} »$. Pertanto, era necessario che l'uso della lingua fosse tutelato dalla mancanza di forma e dalla semplificazione. Inoltre, a proposito dell'imbarbarimento dello spirito linguistico, Kraus riprendeva la critica di Schopenhauer contro il decadimento della struttura formale della lingua. In questa prospettiva, l'insegnamento di un uso corretto del linguaggio, che salvaguardasse la lingua da ogni possibile attacco dello Zeitgeist, diventò per Kraus, come già per il filosofo del Mondo, un interesse continuo ed insistente, che negli articoli della «Die Fackel ${ }^{19}$ » si mostrò come un vero e proprio punto nodale.

Tant'è vero che nel giugno del 1921 uscì con la sua rivista un inserto proprio sull'insegnamento linguistico. Inserto, che il giornalista austriaco intendeva pubblicare in seguito come scritto autonomo che avrebbe dovuto recare come titolo La lingua, ma che non fu mai pubblicato.

Imbevuto, per così dire, di uno schopenhauerismo disincantato e mai dogmatico, Karl Kraus acuì ancor più la sua critica al malcostume letterario del tempo, polemizzando sempre con lo stesso atteggiamento caustico e pungente che lo caratterizzava.

Nel suo saggio sullo stile, Schopenhauer aveva evidenziato l'esistenza di ben tre generi differenti di scrittori, ossia quelli che scrivono senza pensare, quelli che mentre scrivono pensano e, infine, coloro i quali prima di scrivere hanno pensato. Solo chi scrive dopo aver riflettuto, per Schopenhauer, può dare una forma letteraria alle sue considerazioni. E sono proprio questi ultimi i più rari tra gli scrittori ${ }^{20}$.

Dal canto suo, Kraus distingueva due classi di scrittori, ossia coloro che lo sono davvero e coloro che non lo sono: la differenza che intercorre fra l'una e l'altra categoria è simile al rapporto che intercorre tra materia e forma; d'accordo con l'idea di Schopenhauer, secondo cui, il valore di un libro risiede nei pensieri dell'autore (materia) e nella capacità dello scrittore di esprimerli (forma) ${ }^{21}$, Kraus

\footnotetext{
${ }^{17}$ Cfr. B. Adamy, Schopenhauer's Rezeption bei Karl Kraus, cit., p.90.

${ }^{18}$ Id., cit., p.91.

${ }^{19}$ Id., cit., p.94.
}

${ }^{20}$ A. Schopenhauer, Sul mestiere dello scrittore e sullo stile, cit. pp. 19- 20. Scrive Schopenhauer: «Si può dire, ancora, che vi siano tre tipi di autori: in primo luogo, coloro che scrivono senza pensare. Questi scrivono ciò che a loro detta la memoria, in base a reminiscenze, oppure attingono, perfino, direttamente da libri altrui. Questa classe di scrittori è la più numerosa.- In secondo luogo, vi sono scrittori che, mentre scrivono pensano. Essi pensano al fine di scrivere. Simili tipi s' incontrano molto spesso.- In terzo luogo, vi sono scrittori che hanno pensato prima di accingersi a scrivere. Scrivono soltanto perche hanno pensato. Sono rari. Lo scrittore della seconda specie, quello che rimanda di pensare finché non si mette a scrivere, può essere paragonato al cacciatore che va a caccia a casaccio: difficilmente riuscirà a portare a casa un gran che. Invece, il modo di scrivere dello scrittore della terza e rara specie somiglierà a una battuta di caccia, per la quale la selvaggina fu prima acchiappata e racchiusa per poi sciamare in massa dalle serve in altro spazio, anch'esso recinto, dove non può sfuggire al cacciatore; di modo che ora gli resta solo da mirare e sparare (l'esposizione). Questa è la caccia più redditizia».

${ }^{21} I d$., cit., p. 25. 
sosteneva quanto segue:

Nel primo caso materia e forma si appartengono come anima e corpo; nel secondo caso materia e forma stanno insieme come corpo e vestito ${ }^{22}$.

Tuttavia, mentre la critica di Schopenhauer intendeva fornire le regole di una estetica del linguaggio, quella di Kraus fu, soprattutto, una denuncia della sottomissione della letteratura della sua epoca alle leggi di domanda ed offerta del mercato. Per Kraus, cioè, la lingua era utilizzata come strumento politico di persuasione dell'opinione pubblica e della società civile, e gli scrittori erano da lui considerati mercenari del potere, e quindi solo pennivendoli alla mercé del potere politico di turno. Proprio costoro con la loro sottomissione al potere, rendevano la cultura corrotta e falsa.

Schopenhauer stesso aveva espresso un giudizio pressoché analogo circa la letteratura a lui contemporanea. Infatti, il Saggio di Francoforte aveva giudicato quella del suo tempo una cultura succube delle mode e del gusto imperante, corrotta a tal punto che gli scrittori finivano per svolgere il ruolo di artigiani di professione, anziché quello di artisti per vocazione.

Lo stato deplorevole della letteratura odierna in Germania e fuori della Germania ha alla sua radice il fatto che si guadagni denaro scrivendo libri. Chiunque abbia bisogno di denaro si mette a tavolino e scrive un libro, e il pubblico è così stupido da comprarlo. Conseguenza secondaria di ciò è la corruzione della lingua. Una grande quantità di cattivi scrittori vive unicamente della stoltezza del pubblico[... $]^{23}$.

Sempre sullo scritto Sul mestiere dello scrittore e sullo stile Schopenhauer aveva dichiarato:

Le riviste letterarie dovrebbero alzare una diga contro lo scribacchiare senza scrupoli del nostro tempo e il conseguente diluvio sempre crescente di libri inutili e brutti, giudicandoli in modo giusto e severo, senza lasciarsi corrompere, flagellando senza pietà ogni compilazione di mestieranti senza vocazione, ogni scritto mediante il quale le teste vuote cercano di soccorrere le tasche vuote, dunque forse i nove decimi di tutti i libri, combattendo in tal modo doverosamente contro i pruriti letterari e le ciurmerie, invece di secondarli facendo combutta con la loro infame tolleranza con l' autore e l' editore al fine di rubare al pubblico tempo e denaro ${ }^{24}$.

Seguendo Schopenhauer, in una glossa intitolata Grammatici ${ }^{25}$ apparsa nella sua «Fackel», Karl Kraus aveva esortato le riviste letterarie del tempo a prendere una dura posizione contro il degrado e la corruzione degli intellettuali, ovvero contro il crescente numero di libri scritti da «mestieranti senza

\footnotetext{
${ }^{22}$ Cfr. B. Adamy, Schopenhauer's Rezeption bei Karl Kraus, cit., p. 102.

${ }^{23}$ A. Schopenhauer, Sul mestiere dello scrittore e sullo stile, cit., p. 18.

${ }^{24}$ Id., cit., pp. 32- 33 .

${ }^{25}$ Cfr. B. Adamy, Schopenhauer's Rezeption bei Karl Kraus, cit., p.96.
} 
vocazione ${ }^{26} \gg$.

Entrambi i pensatori sostenevano, seppur in tempi diversi, che si facesse un uso improprio della libertà di stampa, causato soprattutto dalla pratica dell' anonimato. Questa pratica era stata legittimamente introdotta per tutelare l'autore, che volesse esprimere giudizi contrari o sfavorevoli al potere, da ogni possibile ritorsione.

Schopenhauer invece riteneva il ricorso all'anonimato solo un mezzo per non assumersi la responsabilità di ciò che si scriveva.

La libertà di stampa, ottenuta finalmente in Germania e di cui si è fatto subito abuso nel modo più indegno, dovrebbe essere almeno condizionata dal divieto di ogni anonimato e pseudoanonimato, affinché ognuno, di quello che annunzia pubblicamente servendosi del portavoce influenze della stampa, sia responsabile almeno sul proprio onore, qualora ne abbia, e, se non ne ha affatto, affinché il suo nome neutralizzi il suo discorso ${ }^{27}$.

Analogamente Kraus, nella sua rivista, rivolgeva un appello diretto al lettore, esortandolo a diffidare dall'anonimato.

\begin{abstract}
Gentile lettore, tu che consideri ancora il giornale come dotato di un potere misterioso, come espressione di saggezza; tu che ti senti attratto dalle curiose rivelazioni a cui non puoi resistere, il tuo rispetto è donato solo all'anonimato. Lascia che il giornalista si sfoghi, come ambasciatore di novità e mediatore commerciale, ma contesta: il suo parlare come da un pulpito e la sua pretesa di esser come stato nominato tanto da poter esprimere valori morali. Non rispettare la parola stampata come se fosse verità ${ }^{28}$.
\end{abstract}

Nel suo capolavoro letterario, Gli ultimi giorni dell'umanità ${ }^{29}$, che Kraus pubblicò a episodi sulla «Fackel» dal 1915 al 1922, egli descrisse con grande esperienza il dramma della Grande Guerra. Questo scritto gli valse la fama di uno dei più grandi scrittori del suo tempo e sicuramente di grande storico di lingua tedesca. In quest'opera Kraus analizzò, attraverso una cronaca sobria e dettagliata, gli eventi storici dell'epoca, dall'assassinio di Sarajevo dell'erede al trono, fino al crollo della monarchia asburgica.

Sempre fedele al metodo storico, egli riuscì a ricostruire e a documentare questo evento drammatico, facendo ricorso a ritagli di giornale, cartoline postali, lettere, volantini e semplici discorsi ascoltati per le strade di Vienna. Inoltre, accanto all'analisi storica degli eventi, non potendo far uso di

\footnotetext{
${ }^{26}$ A. Schopenhauer, Sul mestiere dello scrittore e sullo stile, cit., pp. 32- 33.

${ }^{27}$ Id., cit., p. 36.

${ }^{28}$ Cfr. B. Adamy, Schopenhauer's Rezeption bei Karl Kraus, cit., pp. 96- 97.

${ }^{29}$ K. Kraus, Gli ultimi giorni dell'umanità, trad. it. E. Bram e M. Carpitella, Milano, Adelphi, 1980.
} 
personaggi reali poiché le leggi asburgiche punivano severamente la rappresentazione di persone viventi, Kraus propose una sua interpretazione della guerra, immaginando tutta una serie di discorsi apocalittici tra l'amante della guerra e l'ottimista, per condannare, infine, il generale entusiasmo per la guerra e la dilagante corruzione della classe politica. Egli descrisse il conflitto come espressione terrena dell'inferno, dove bambini trasportano cadaveri e Dio dice: «Io non ho voluto questo ${ }^{30} »$.

La scrittura storica di Kraus testimonia uno scetticismo di fondo e un pessimismo che non sfociano però in alcun sistema filosofico.

Sempre in questo scritto, seppur senza riferimenti precisi al filosofo, Kraus polemizzava contro la metafisica del pessimismo del Mondo come volontà e rappresentazione, ritenendo che non occorresse nessuna fondazione metafisica del pessimismo, poiché questo finisce per manifestarsi "realmente" e tangibilmente nella disperazione e nella miseria umane.

Da storico ed osservatore del suo tempo, Kraus prese le distanze dalla prospettiva antistoricistica del filosofo di Danzica, il quale considerava la storia non una scienza ma un sapere ${ }^{31}$.

Se Schopenhauer aveva trovato nella contemplazione estetica del genio una via, per quanto effimera, di redenzione dal pessimismo (metafisico), Kraus invece scrutava a fondo il microcosmo della società viennese e sosteneva una forma di pessimismo antropologico, fondato proprio su quelle "osservazioni psicologiche" dei vizi e delle virtù umane indicate da Schopenhauer nei Parerga $e$ paralipomena, colte però nella loro dimensione storica e sociale.

In conclusione, quella del pensiero di Schopenhauer su Kraus non è una influenza limitata esclusivamente alla teoria estetica o allo stile linguistico, bensì essa si manifesta più profondamente in un atteggiamento intellettuale ed in una visione del mondo al contempo pessimistica e disincantata. Di Schopenhauer Kraus rifiuta certamente il lato metafisico ed antistoricistico, ma accetta e condivide il primato romantico del genio e sviluppa il lato illuministico di Schopenhauer, quello del saggista e del moralista.

\section{Nota bibliografica}

ADAMY, B. Schopenhauer’s Rezeption bei Karl Kraus. In: «Schopenhauer Jahrbuch», 1985.

FISCHER, E. Karl Kraus, Robert Musil, Franz Kafka. Presentazione di L. Lombardo Radice. Firenze: La Nuova Italia 1974.

\footnotetext{
${ }^{30}$ Cfr. B. Adamy, Schopenhauer's Reseption bei Karl Kraus, cit., p. 86.

${ }^{31}$ A. Schopenhauer, Il mondo come volontà e rappresentazione, cit., p. 1825. Scrive Schopenhauer:« La scienza scevera il molteplice innumerevole, lo raccoglie sotto concetti di genere, e questi a loro volta sotto concetti di specie, aprendo in tal modo via ad una conoscenza del generale e dello speciale che abbraccia le innumerevoli cose particolari, in quanto vale di tutto, senza che si debba considerare ogni cosa per sé »».
} 
KRAUS, K. Gli ultimi giorni dell'umanità, trad. it. E. Bram e M. Carpitella. Milano: Adelphi, 1980. . Aforismi, a cura di P. Sorge. Firenze: Sansoni Editore 1992.

SCHOPENHAUER, A. Parerga e paralipomena, a cura di G. Colli. Milano: Adelphi, 1981. . Sul mestiere dello scrittore e sullo stile, trad. it. E. Amendola Kuhn, con una nota di F. Volpi. Milano: Adelphi, 1993.

TIMMS, E. La Vienna di Karl Kraus. Bologna: Il Mulino, 1986.

Recebido: 30/05/12

Received: 05/30/12

Aprovado: 21/12/12

Approved: 12/21/12 